\title{
Intravitreal ganciclovir in CMV retinitis: case report
}

\author{
M L HARRIS AND $M$ B R MATHALONE \\ From St Stephen's Hospital, Fulham Road, London SW10 9TH
}

SUMMARY A case is reported of a 35-year-old male with established acquired immune deficiency syndrome who developed cytomegalovirus retinitis. When intravenous treatment with ganciclovir had to be withdrawn because of toxicity, one eye was treated successfully by intravitreal injections of the drug. After 15 weeks of intravitreal treatment this eye developed endophthalmitis.

A frequent ophthalmological complication of the acquired immune deficiency syndrome (AIDS) is cytomegalovirus (CMV) retinitis. ${ }^{14}$ Until recently this blinding condition was untreatable. However, the acyclovir analogue ganciclovir given intravenously has been shown to be effective in CMV retinitis, ${ }^{5}$ with an $88 \%$ response rate. ${ }^{6}$ There are disadvantages to its use. Firstly, it is a virostatic agent and maintenance treatment is required ${ }^{6-8}$ Secondly, it is toxic, and neutropenia occurs in up to $38 \%$ of patients on maintenance treatment. ${ }^{89}$ Thirdly, 'breakthrough' disease is encountered on maintenance therapy in up to $50 \%$ of patients. ${ }^{8}$ We report on a patient who has had intravitreal injections of ganciclovir when systemic treatment had to be abandoned owing to neutropenia.

\section{Case report}

A 35-year-old male homosexual with established AIDS presented in March 1987 with painless loss of vision in the left eye of three days' duration. Ocular examination showed a corrected visual acuity of $6 / 5$ right and 6/9 left, with extensive loss of temporal visual field in the left eye. Biomicroscopy revealed flare in the aqueous of the left eye, and on funduscopy there were the typical changes of CMV retinitis involving the nasal retina of the left eye up to and surrounding the optic nerve head. In addition there was a haemorrhagic patch of retinitis just inside the inferior temporal arcade of the right retina 2-3 disc diameters in area (Fig. 1).

The patient was admitted to hospital and started on intravenous ganciclovir $5 \mathrm{mg} / \mathrm{kg}$ daily. On admission his white cell count was $1.8 \times 10^{9} / 1$, and rectal biopsy showed mild proctitis with CMV inclusion particles.

Correspondence to M L Harris, FRCS.
After an initial decline in the vision of the left eye the disease came under control, and maintenance treatment of $5 \mathrm{mg} / \mathrm{kg}$ on three days per week was started. During this period his white cell count fell, the dosage of maintenance treatment was halved, but after 12 weeks of treatment his white cell count had fallen to $0.8 \times 10^{9} / 1$ and intravenous ganciclovir had to be discontinued. His visual acuity at this time was $6 / 12$ right, $6 / 24$, left.

We decided to treat the right eye by intravitreal injections of ganciclovir. Injections of $200 \mu \mathrm{g}$ ganciclovir in $0.1 \mathrm{ml}$ of normal saline were prepared in a sterile cabinet and passed through a Micropore filter giving a near maximum soluble solution of $\mathrm{pH}$ $10 \cdot 14$. After topical anaesthesia with amethocaine $1 \%$ drops the injections were made through the inferior pars plana via a 27 gauge needle on a tuberculin syringe. The circulation at the optic nerve head was checked after each injection; we did not find it necessary to perform an anterior paracentesis. Topical chloramphenicol $0.5 \%$ drops were given four times a day, on the days preceding and following as well as on the day of each injection. The treatment was given twice weekly for three weeks and weekly thereafter. The injections were generally well tolerated. The retinitis in the right eye showed a good response to this treatment while the retinitis in the left eye showed rapid progression. By the thirteenth week of intravitreal therapy the visual acuity of the untreated left eye was hand movements, that of the right eye remained at $6 / 12$, but funduscopy showed new haemorrhages and exudates extending on both sides of the fovea (Fig. 2).

After 15 weeks of intravitreal treatment (total dose $30 \mathrm{mg}$ ) the patient returned to the clinic with a painful, red right eye. He was admitted to hospital for treatment of a right endophthalmitis. A specimen from diagnostic vitreous biopsy grew a mixture of two 


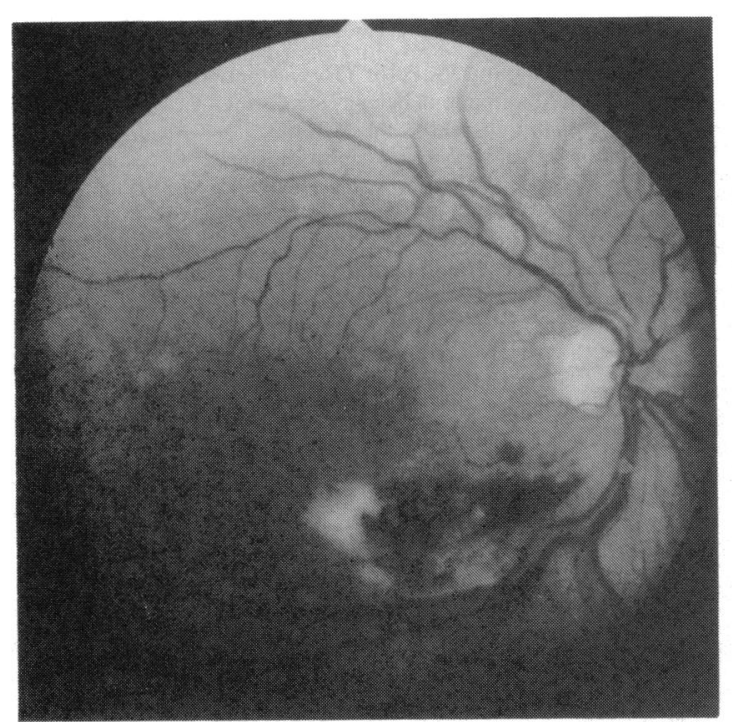

Fig. 1A

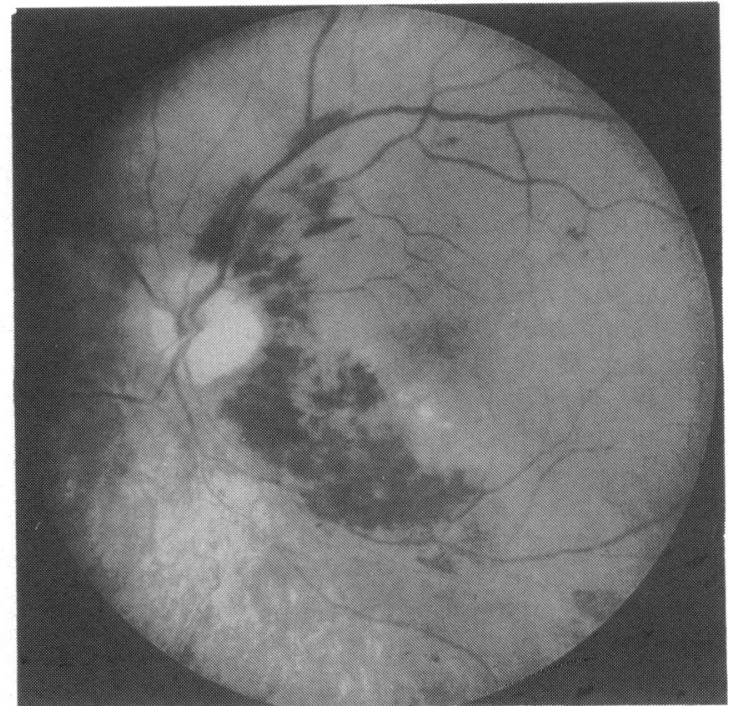

Fig. 1B

Fig. 1A, B Right and left fundi at presentation, showing active retinitis in both eyes.

coagulase-negative staphylococci, both resistant to chloramphenicol and one also to methicillin. His endophthalmitis responded to treatment, the vision recovering from perception of light to counting fingers. Concurrently he had developed a recurrent pneumocystis pneumonia and died 18 days later. Permission for post-mortem examination was not obtained.

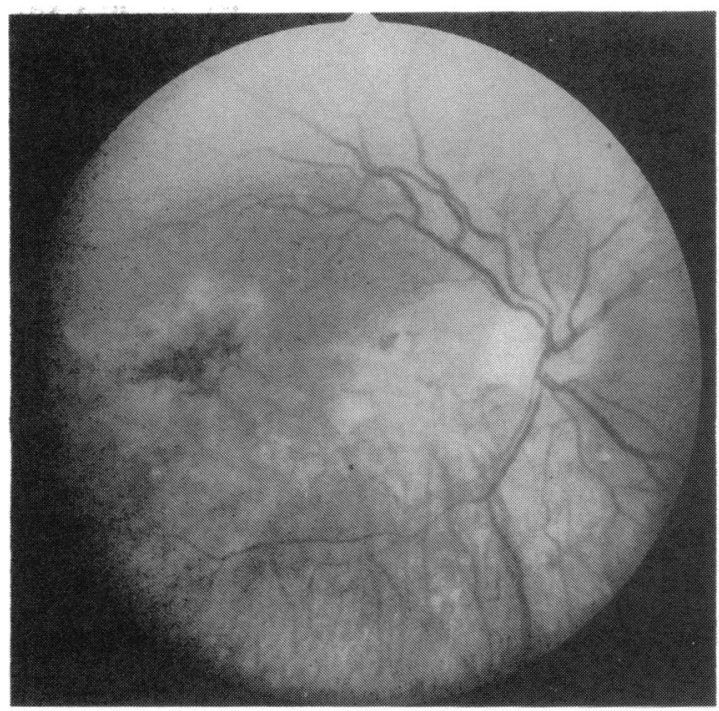

Fig. 2A

\section{Discussion}

In 1985 Pulido et al. ${ }^{10}$ found no ophthalmoscopic or histological changes on the retina after single-dose injections of ganciclovir into rabbit vitreous. Henry et al. " have reported the use of this agent via intravitreal injection in one case of CMV retinitis. Using an $\mathrm{ID}_{50}$ (dose sufficient to reduce viral replication to

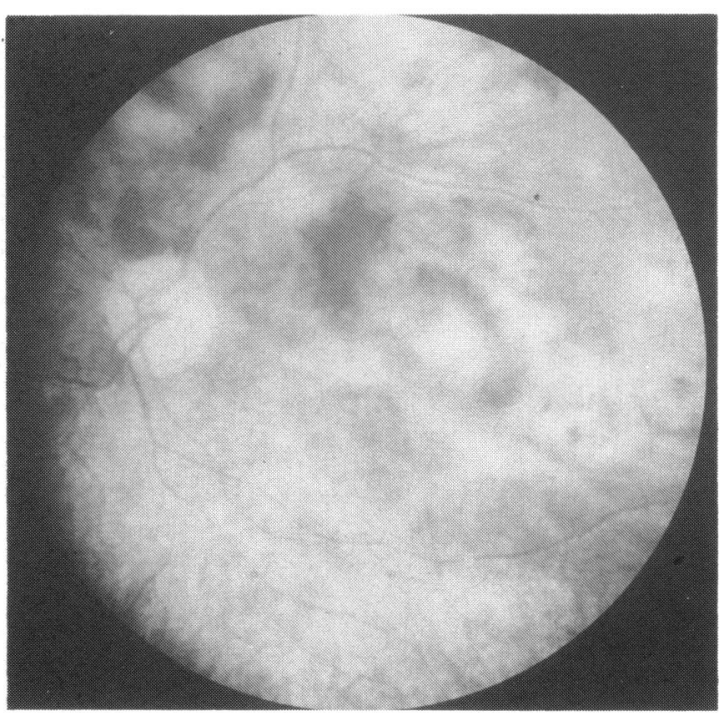

Fig. 2B

Fig. 2A, B Right and left fundi after 14 weeks of intravitreal ganciclovir to the right eye. Note active edge of retinitis on both sides of the right fovea (A) and widespread destruction of left retina with secondary optic atrophy (B). 
$50 \%$ ) value of $2.87 \mu \mathrm{g}$ Henry et al. ${ }^{.}$calculated that the intravitreal concentration of the drug remained above this level for approximately 62 hours after a single intravitreal injection of $200 \mu \mathrm{g}$. They were able to control the retinitis in their patient with weekly injections. Our case confirms that intravitreal administration of ganciclovir can be used as an alternative route in cases where the use of intravenous therapy is limited by the occurrence of leucopenia. The late recurrence of disease raises the question of whether maintenance treatment of weekly $200 \mu \mathrm{g}$ is sufficient to control the retinitis adequately. A larger dosage may be desirable, but $200 \mu \mathrm{g}$ in $0.1 \mathrm{ml}$ is close to the maximum solubility for the drug. Injecting larger volumes intravitreally would certainly require anterior paracentesis to prevent increased intraocular pressure. It remains to be proved that repeated injections of this alkaline agent are not retinotoxic.

This case was complicated by the endophthalmitis, which was always a possibility in this situation. Staphylococci are the commonest cause of endophthalmitis ${ }^{13}$ and are remarkable for their ability to develop resistance to antibiotics. This makes the choice of prophylactic agents difficult.

In conclusion, ganciclovir has been found to be an effective agent when used intravitreally in CMV retinitis. A maintenance dose of $200 \mu \mathrm{g}$ weekly does not exclude the possibility of breakthrough of the disease. Endophthalmitis is a constant threat when repeated intravitreal injections are given.

\section{References}

1 Holland GN, Pepose JS, Pettit TH, Gottlieb MS, Yee RD, Foos RY. Acquired immune deficiency syndrome - ocular manifestations. Ophthalmology 1983; 90: 393-402.

2 Khadem M, Kalish S, Goldsmith P, et al. Ophthalmologic findings in acquired immune deficiency syndrome. Arch Ophthalmol 1984; 102: 201-6.

3 Palestine A, Rodrigues M. Macher A. et al. Ophthalmic involvement in acquired immunodeficiency syndrome. Ophthalmology 1984; 91 : 1092-9.

4 Mines J, Kaplan H. Acquired immunodeficiency syndrome (AIDS): the disease and its ocular manifestations. Int Ophthalmol Clin 1986; 26: 73-115.

5 Palestine A. Stevens G, Lane H, et al. Treatment of CMV retinitis with dihydroxypropoxymethyl guanine. Am J Ophthalmol 1986; 101: 95-101.

6 Holland G. Sidikaro Y, Kreiger A, et al. Treatment of cytomegalovirus retinopathy with ganciclovir. Ophthalmology 1987; 94: $815-23$

7 Harris M, Mathalone M. Dihydroxypropoxymethyl guanine in the treatment of AIDS related retinitis due to cytomegalovirus. Br Med J 1987; 294: 92.

8 Jabs D, Newman C, de Bustros S, Polk BF. Treatment of cytomegalovirus retinitis with ganciclovir. Ophthalmology 1987: 94: 824-30.

9 Henderly D. Freeman W, Causey D, et al. Cytomegalovirus retinitis and response to therapy with ganciclovir. Ophthalmology 1987: 94: 425-34.

10 Pulido J, Peyman G, Lesar T, Vernot J. Intravitreal toxicity of hydroxyacyclovir (BW-B759U) a new antiviral. Arch Ophthalmol 1985: 103: 840-1.

11 Henry K, Cantrill H, Fletcher C, Chinnock BY, Balfour HH. Use of intravitreal ganciclovir for cytomegalovirus retinitis in a patient with AIDS. Am J Ophthalmol 1987; 103: 17-23.

12 Forster RK. Postoperative endophthalmitis; etiology and diagnosis. Ophthalmology 1978: 85: 320-6.

13 Puliafito CA, Baker AS, Haff J, et al. Infectious endophthalmitis. A review of 36 cases. Ophthalmology 1982; 89: 921-9.

Accepted for publication 18 July 1988. 